\title{
Reassessing the Methods of Foreignizing and Domesticating Translation
}

\author{
Xinkan Zhao \\ Department of Philosophy, Peking University, China
}

\begin{abstract}
This paper clarifies the frequently used concepts of foreignizing and domesticating translation to the extent that they can be meaningfully evaluated. As it turns out, foreignizing translation is best understood as including more than one method, with each having its own evaluative profile, and domesticating translation should be cleared of confusion with other methods that have historically brought it a bad name. Based on detailed evaluations, the paper briefly proposes collaboration as the proper way forward and also shows how the ambitious strategy of foreignizing, which might be named after the German Romantic scholar Friedrich Schleiermacher, is special and what reasons there are not to accept it. Before concluding, the paper addresses issues most relevant to translating philosophical texts, where terminologies have often already been foreignized through stipulation.
\end{abstract}

Index Terms-methods of translation, foreignizing translation, domesticating translation, philosophical translation, Friedrich Schleiermacher

\section{INTRODUCTION}

In translation theory, the distinction between "foreignizing translation" and "domesticating translation" is a popular one. It is also often presented in different forms, such as translating "strictly" versus "loosely", translating "word-for-word" versus "sense-for-sense", "moving the reader to the author" versus "moving the author to the reader", and so on. But whichever form it takes, neither side of the distinction seems to have received clear definition or explication other than vague descriptions that try to capture the general feature. In this line, it is often said that foreignizing translation is the method that stays faithful to the language of the source text, resulting in a translation that looks strange and foreign, while domesticating translation is the method that emphasizes requirements of fluency and clarity in the target language, which makes the author appear native. But clarity is severely lacking in such descriptions if serious evaluations of the respective methods are to be made.

The task of this paper is above all to clarify the concepts of foreiginizing and domesticating translation as formally as possible (Section 2), and to fill out their evaluative profiles respectively (Sections $3 \& 4$ ). Based on such evaluations, collaboration between them is proposed as the way forward (Section 5) and reasons are given to show how an ambitious form of foreignizing translation, attributable to Friedrich Schleiermacher, is special and what reasons there are not to accept it (Section 6). Before concluding, attention is paid to issues most relevant to translating philosophical texts, where terminologies have already been foreignized through stipulation (Section 7).

\section{FRAMEWORK AND ANALYSIS}

To arrive at the clear concepts of foreignizing and domesticating translation, it is useful to stipulate three "naturals" as foundations for following explanation: natural semantic content, natural expression, and natural usage. Here and throughout, "natural" is used in the sense of "native", and "foreign" or "unnatural" as its antonym.

A natural semantic content in a linguistic community is one that people in that community understand and express with linguistic devices comfortably, without intellectual pressure. While arguably all human beings share largely the same spectrum of semantic content since they have largely identical physiological (esp. neural) structures in an evolutionary sense, different communities are sure to have different natural semantic contents due to historical contingencies. Intuitively, different portions of the ultimate spectrum of intelligibility are highlighted in different communities. Typically a natural semantic content in one community may be either natural or foreign in another.

A natural expression is a linguistic device, which is often a string of words rather than a single word, commonly used in the community. They are the expressions that people in the community do not find surprising merely by their look (or sound, in the case of oral language), and they are the ones to be expected in a well-composed local dictionary.

This is not to be confused with a natural usage of an expression. While the former is exclusively concerned with the syntactic features of the expression, this notion attends to how an expression is connected to a semantic content. Call a usage of an expression "natural" if the expression is intended to express the semantic content that people in the community typically and comfortably associate it with.

It is essential to note that while the standard case is where natural expressions are naturally used to express natural semantic contents, there are other possibilities. For example, a natural expression can be unnaturally used for some unnatural semantic content; an unnatural expression can be unnaturally used for some natural semantic content people 
already have a natural expression naturally used for; and so on.

This much preparatory work done, it is time to formulate what the central distinction amounts to. Foreignizing translation, then, is simply the way of translation that, when faced with foreign semantic contents, employs at least one unnatural element, either unnatural expressions or unnatural usages of expressions, or both. And domesticating translation amounts to the way of translation without resorting to any unnatural elements: both expressions and usages must be natural.

Note that, on this construal, foreignizing translation takes place only if there is foreign semantic content, which is also intuitively correct. For why bother introduce foreign elements if the semantic content is already natural to the target community, (that is, if there is already a natural expression to express it without being used unnaturally) making domesticating translation the apparently undisputable option? But this is not agreed by all, especially ambitious foreignizing translators, whom will be discussed in due course; for the time being, the arguments stick to the more commonsensical.

\section{Methods of Foreignizing Translation}

As is implied, the method (purportedly singular) of foreignizing translation is in fact a collection of more than one: to express the foreign semantic content, the translator may either (i) use natural expressions unnaturally, or (ii) use unnatural expressions naturally, or (iii) use unnatural expressions unnaturally. Presumably the third option exists only as a theoretical combinatory possibility, and only the first two, which are clearly discoverable in practice, deserve a closer look.

The first option might be dubbed as "bending", as is suggested by Johann Herder, another Romantic scholar, who adopts and praises this method. Chapter 12 of Foster (2010) gives a detailed example explaining how it works. ${ }^{1}$ Here is a brief restatement of the example: take the Greek word "arete" and the closest counterpart in English "virtue". Despite their similarity in referring to a kind of excellence, they are not the same. For example, "arete" applies to pirates or habitual liars, but "virtue" does not; conversely, "virtue" applies to virtuous men who are physically weak, but "arete" does not. What does the method of bending do? It simply uses "virtue" in a way as if it just were "arete". The translator "bends" the word so that it picks up unnatural usages (allows talks about pirates of virtue) and avoids certain previously natural usages (stops talking about virtuous cripples). "Virtue" now looks like "arete" in disguise, hence an instance of "foreignization".

How is such a method to be evaluated? It is said to have the advantages of achieving faithfulness to the source text and enriching the target language. ${ }^{3}$ But each claim needs to be examined more closely. First, for the faithfulness claim, if "faithfulness" means the preservation of semantic content expressed in the source language, then this achievement should be acknowledged, for after the expression is bent to the foreign shape, it does indeed preserve the original semantic content, since the semantic content of the bent expression just $i s$ that of the original expression, by the very definition of bending! But such preservation requires the extra care on the part of the reader to keep in mind that whatever is presented on the page might have been bent to mean something different. In this sense, the best one can say is that the preservation of content is what the method is capable of but not its advantage.

What about the alleged advantage of enriching the target language? Consider again the example of "arete". It might be argued that, through bending, the translation enriches English since now "virtue" can mean what the Greek word "arete" means, a semantic capability that was missing previously, hence enrichment. But this argument seems to overlook what happens to the original English word "virtue", the naturally used expression. If the linguistic community were to reserve "virtue" to express what Greeks would with "arete", wouldn't they need another English word for what was originally expressed by the English "virtue"? And further, if they bent this other word to fill in the slot, wouldn't they need yet another word to express what was originally expressed by this other word? So on and so forth. In this case, it's more accurate to say that such translation shifts English semantics rather than enriches the language.

Alternatively, the proponent might argue that bending does not overwrite the original semantics of the English word, but rather adds to it, so that "virtue" can now mean both what was originally meant by this word and what "arete" means in Greek. But then this would be a case of generating ambiguities rather than enrichments. Perhaps the proponent might want to say that adding a new meaning to the original word is a form of "synthesis", so that one can now see the English virtue and Greek arete are aspects of a larger concept which is now signified with "virtue", hence an enrichment. But this does not seem to be a case of synthesis, for in synthesis one needs to be able to discern what have been synthesized into this larger concept, while now the speaker is in a complete lack of resources to denote the sub-concepts. So this is more a case of conflation than that of synthesis.

The above arguments show that bending, the first method of foreignizing translation, does not have as many advantages as it seems. What's worse, it might suffer from quite a few disadvantages. The most severe problem is the one already touched upon above and complained by many: by either shifting or conflating semantics of expressions, this method badly impairs the readability of the translation. Indeed, sometimes it wouldn't be an exaggeration to call the

\footnotetext{
${ }^{1}$ Foster (2010). The example appears on pages 399-400.

2 Note that "being the closest" does not have much bearing on the demonstration this paper is currently concerned, as long as the Greek word expresses a semantic content foreign to English.

3 As in Foster (2010), p397.
} 
resulting language a third language, distinct from both the source and target. Reading becomes deciphering not without much guesswork. This problem is probably best summarized in famous line by Yan Fu, the $19-20^{\text {th }}$ century Chinese educator and translator:

"If, with faithfulness attended to, expressiveness is not achieved, then such translating is much like not having translated anything."4

Moreover, the low level of readability can make the source content appear mystic, which may in turn foster unwanted attitudes among the non-academic readers towards such content. Positive attitudes may evolve into cult following, and negative ones may transmute into resentment and disgust, both of which are irrational and inappropriate. ${ }^{5}$ Translation, a noble task that aims at bridging cultures, is thus threatened if not undermined.

Finally, this method, especially if applied widely, causes practical troubles for publishers: it makes quality check much harder, for there is no reliable standard to distinguish between a bent usage and a misusage. What is the publisher to tell a translator who submits a translation full of ungrammatical expressions but insists on their being not only correct and truthful, but also deep and insightful, on grounds that those are merely bent expressions which look disorganized only to the untrained eyes? One cannot reject such work for its unnaturalness, for this feature is perfectly allowed by the method.

Having said these, it might be tentatively concluded that the prospects for bending look bleak, and the second option seems to be calling for attention, namely, to express the foreign semantic content with an unnatural expression used naturally. It may appear odd at first sight to talk about natural usages of unnatural expressions, but no longer so if one sticks to the definition. An expression is naturally used if it is intended to express what people in that community typically and comfortably associate it with, so even though an expression is unnatural, it can still be naturally used as long as it expresses what people typically and comfortably associate it with. For example, the expression "deforeignization" may not be expected in a normal dictionary but one can say, without oddity, that it naturally means "the process that makes something less foreign". Call this foreignizing method "neologism".

The profile of neologism is overall better than bending. First, neologism preserves the original semantic content just as well as bending does, but it has the advantage of not needing the readers' extra caution. Second, it genuinely enriches the target language, for it introduces new words instead of either overwriting old ones or getting conflated with them; the old semantics remains unaltered. And for this very reason, third, neologism may lower readability but the problem is much less serious than it is with bending, and fourth, quality check is easier for publishers, for one can decide its quality by seeing if associating the expression with the foreign content turns out easy.

The biggest difficulty for the method of neologism is rather the very task of finding the new expression itself. While there are almost always several candidate expressions extant in the vicinity that a translator can bend, it is much harder to coin a new expression that can naturally lead the reader to the foreign content, and even if it does, it may also sometimes lead the reader to other semantic contents equally naturally, thus making the new expression ambiguous from the start. Perhaps a good way out is to simply import the entire original expression, leaving it untranslated. ${ }^{6}$ But translating by leaving as translated is better seen as a last resort, which suggests that other possibilities are worth exploring.

\section{DOMESTICATING TRANSLATION}

Domesticating translation is often characterized as the way of translating that makes the target text look like what the original author would have written had the author been capable of the target language. ${ }^{7}$ This counterfactual formulation is popular among the translators who are supportive of fluency in translation, but it receives vehement attack from Schleiermacher, who believes such a method is "null and void in itself". 8 He bases his argument on the impossibility of separating an author from the author's first language, owing to the essential shaping power of language on any human being. Now that such tearing apart is a conceptual impossibility, the counterfactual formulation fails as a nonstarter. He presents much scorn and sarcasm in part of his formulation of the argument:

"Indeed, what can be the objection if a translator were to tell a reader: Here is the book just as the author would have written it if he had written in German, and if the reader were to reply: I am much obliged to you, in the same way as if you had brought me the picture of the author just as he would have looked if his mother had conceived him with another father? For if the writer's particular spirit is the mother of works belonging to scholarship and art in a higher

\footnotetext{
${ }^{4}$ Yan (1931). See his Notes on Translation. (My translation, same below.) Yan Fu as an important English-Chinese translator was also interestingly famous for violating his own theorizing when actually translating, which will be addressed in more detail shortly.

${ }^{5}$ Interestingly, positive and negative attitudes may well feed on each other in a self-reinforcing pattern: the resentment and disgust greatly fuels the cult, which in turn aggravates the former. This phenomenon is discernible at least in some of the practices translating western literary works into Chinese.

${ }^{6}$ See for example Hansen (1992), which simply renders the Chinese term “道” into its pinyin form, "Dao", and Frege (1997), which leaves the German word "Bedeutung" untranslated, though each may have based the choice of translation on other reasons than those under discussion here.

7 A close but subtly different version, applicable only to texts with characters that speak out lines, is to translate in a way that the character would have spoken, had that character been able to speak the target language. For example, when Martin Luther was defending his way of translating, he said, "because this is what [the angel] is trying to say and this is how he would have said it, if he had wanted to greet her in German." See Weissbort \& Eysteinsson (2006) p62.

${ }^{8}$ Schleiermacher (1813), p21.
} 
sense, his national language is the father."

But despite the argument's power in establishing the impossibility of changing an author's first language, one should be careful not to equate this with a refutation of the possibility of domesticating translation. As has been defined previously, domesticating translation is type of translation that expresses foreign semantic contents in the source text using natural expressions in a natural way. But this does not at all require one to imagine any author to be thus-and-so; what is at work here is no more than the three notions of naturalness, which are further defined by psychological states such as comfort and ease. The fact that the actual author as a person is hardly relevant can be shown by how translation is actually practiced. Suppose the author of a book is unknown, or known to be one of a few people but no evidence can show which. Is the translating process thereby disturbed? It doesn't seem so. Similarly, what if in the middle of translating, the author is confirmed to be someone unexpected, would that affect much of what is going on? Not either. Hence the idea of domesticating translation is at least viable. ${ }^{10}$

The definition given above suggests there is only one method of domesticating translation, namely, to express foreign semantic contents with natural expressions used naturally, however, the term has been historically associated with two other methods, each with a different understanding of either what "semantic content" means or what counts as "expressing" it.

The first method takes the "semantic content" to be the "gist" of the source text. As long as the broader stokes, the theme, the motif, the core idea of the original work is maintained, the translation is considered successful. This method then grants tremendous freedom to the translator, so much so that it allows translation to be restatement. This method is exemplified by Yan Fu's work among others. In his prefatory notes, he wrote:

"The translation clarifies the deep meanings; therefore in between words and sentences, it reorders and restructures every now and then, and does not stick to word-to-word, sentence-to-sentence stricture. However its meaning shall not go against the original text." 11

Interestingly, this method has been criticized even by Yan Fu himself. He does not give an explanation for this self-criticism, but the reason is relatively obvious: that frequently in translation, and more so in scholarly translation, the details at the level of phrases are as important as the "gist" the scholars are concerned with. The method of restatement therefore runs the risk of overlooking important contents, which makes the resulting work an essentially incomplete one.

The second method apparently understands "express" as "more or less express" rather than "strictly, faithfully, or simply, literally express". ${ }^{12}$ This method has the unparalleled virtue of really bringing the author home, but it has serious consequences when translating expressions that carry information revealing fundamental differences between cultures. Call this method "forced ontological mapping", since it maps the foreign semantic contents onto native ones that are close, familiar yet fundamentally different. Imagine a Chinese-to-English translator saying "The 天 [sky, heaven] in the East just is God in the West, so why not use 'God' to translate '天'?"13 This is a typical case of the very many ones where the foreign content is implicitly replaced rather than preserved, hence a failure in translation.

Since this method has an amiable appearance, being fluent and reader-friendly, it may receive a much wider popularity than the previous method, whose error is easier to spot by someone who understands both languages. For this very reason, it may generate more misunderstanding and suppress efficiency in cross-cultural communication, and it well deserves the criticisms that have been made against it. At this point, a careful translator is sure to find Lawrence Venuti insightful in regarding this method of translation as unleashing an "ethnocentric violence of translation" on the source text, producing an "ethnocentric reduction of the foreign text to target-language cultural values". ${ }^{14}$ And this is also where one finds Roger Ames' calling for an "interpretive context" appropriate to the source culture much-needed, for example in translation from (classical) Chinese to English and other western languages, "the vocabulary established [...] has been freighted by an often unconscious Christian framework, and the effects of this 'Christianization' of Chinese texts are still very much with us." 15

These considerations then leave available only the option as suggested by the definition: natural expressions plus natural usages for foreign contents. Intuitively, this method of translation carries with it an analysis or explanation of the original contents, so dub it "translation through explanation". Consider for example Ames' rendering of the classical Chinese “君子” as "exemplary person” or "consummate human being", which can be seen as a product of the domesticating translation policy in this acceptable sense, without mapping it onto something as mundane as

\footnotetext{
9 Ibid, p25.

${ }^{10}$ Sometimes skepticism against domesticating the foreign contents appear under the guise of "radical translation", which have been discussed by important $20^{\text {th }}$-century figures like W. V. O. Quine and Donald Davidson. But this paper doesn't seem to be the right place to confront with such cases, since of all the linguistic communities that are implicitly considered here, no one community is foreign in such a radical sense to any other, all being physiologically similar to, reproductively compatible with and evolutionarily continuous from each other.

11 Yan (1931). Notes on Translation.

12 "Literally" is used here in the sense of "without being rhetoric". It is not intended to mean being inferred from the roots or their combinations within the expressions. So "manage" literally (in the first sense) means "to control, organize or deal with", but it can also be said to literally (in the second sense) mean "to control with hands only". This paper uses the word in the first sense.

${ }_{13}$ Giles (1889) did exactly this. See for example p18.

14 Venuti (1995), p20.

15 Ames (2017). See especially page 6 for a list of examples of inappropriate equivalents in Chinese-English translation.
} 
"gentleman" or as religious as "saint". 16

This method has the advantage of both preserving the original content, since the translation carries explanation with itself, and achieving fluency, due to its compliance with the naturalness constraint. The only problem is that when an expression (especially a word) is translated through explanation, the very fact that certain semantic contents have been crystalized into one single expression in the source community may be overlooked. The reader may get comfortably lost in fluency without realizing an important aspect of how the world has been "carved up" by that other culture, or more simply its "conceptual scheme".

\section{TAKING STOCK}

It is helpful at this point to take stock and see which way forward serious academic translation should adopt. As is shown above, foreignizing translation can be understood in different ways, but only the method of neologism is practically favorable. It enriches the target language only at the minor cost of relatively but acceptably lowering readability, though it must face the challenge that suitable neologisms are hard to come by. Domesticating translation, when cleared of confusions historically associated with it, is one single method which renders the resulting text with much fluency but at the cost of being less informative about target conceptual schemes. Both preserve the original content.

Given these features, it is not difficult to come up with a collaboration proposal. When the expressions to be translated reflect fundamental ways of understanding the world, foreignizing (as neologism) is preferred, and for the rest, domesticating is preferred. This seems also to capture nicely our pre-theoretical intuitions about translation, only that before theorizing a clear articulation of it is far from available.

A practical difficulty that such a combinatory policy may face is to decide which portions of the text do "reflect fundamental ways of understanding the world" and which do not, for many expressions are plainly ordinary but may potentially contribute substantially to philosophizing. For a well-known example, the extremely ordinary expression "to be" can potentially escalate into debates about Being as such. This flexible feature of language makes it impossible for to predetermine where to draw the line; the only solution left with the translator seems to be to decide project by project with prudence.

\section{Against Ambitious Foreignizing Translation}

So far the discussion has been restricted to situations where the translator is faced with foreign semantic content. When no semantic content is foreign, domesticating translation has been assumed to be the default option. But this is not agreed by all, and it's now time to turn to this issue as promised before. According to the idea of an ambitious form of foreignizing translation, the method of foreignizing is to be applied uniformly regardless of the naturalness of source text. (How to foreignize is not touched upon by this debate.) This special form of foreignizing translation might be dubbed "Schleiermacherian foreignizing translation", since it is arguably the advocating of such uniform application of the foreignizing method, namely the scope, instead of the method itself which is ancient, that distinguishes Schleiermacher from the translators before him. In his now-celebrated 1813 lecture "On the Different Methods of Translation", Schleiermacher says,

“...[I]t will have to be admitted that an indispensable requirement of this [reader-to-author] method of translation is a feeling for language that is not only not colloquial, but also causes us to suspect that it has not grown in total freedom but rather has been bent towards a foreign likeness..."17

Here the foreignness is elevated as a purpose (requirement) in itself instead of some unfavorable style that the translator and reader have to put up with. The strangeness of this requirement is immediately admitted by himself when he says it "seems to be the strangest form of humiliation a writer who is not a bad writer could impose upon himself". ${ }^{18}$ But then why advocate such a humiliation? Schleiermacher's answer seems to be so that the translator can "transfer this feeling - that they have something foreign before them - to the readers whom he offers a translation in their mother tongue". ${ }^{19}$ But still, why should the translator be obliged to transfer such a feeling? What's so bad about generating fluency and transparency and not transferring such a feeling, especially if such foreign feeling is not present when the source text is originally presented to its intended reader? Venuti (1995) provides a good answer when addressing this issue, though not without ambiguity:

"Foreignizing translation signifies the difference of the foreign text, yet only by disrupting the cultural codes that prevail in the target language. In its effort to do right abroad, this translation method must do wrong at home, deviating enough from native norms to stage an alien reading experience." 20

The ambiguity lies in exactly what "signifies the difference" means. For it can mean "to signify the fact that there is a difference", and further it can also mean "to signify where the difference consists in". Based on the principle of charity,

\footnotetext{
${ }^{16}$ See for example Ames (2017).

17 Schleiermacher (1813), p16.

18 Ibid, p16.

19 Ibid, p15.

${ }^{20}$ Venuti (1995), p20. Emphasis added.
} 
it is assumed here that the term covers both meanings. So the reason for transferring a foreign feeling consists in the following: (i) it reminds the readers that they are facing a foreign culture; (ii) it helps the readers to see more clearly what the foreign culture is like and how it is different from the target native culture.

But both propositions need a second thought. For the first proposition, despite its truth, there seem to be numerous other more convenient and equally efficient ways of reminding. For example, can't one be easily reminded by directly seeing the foreign culture that is nonetheless fluently and transparently presented? Indeed, one can even be easily reminded by the book cover that states it is a translated work. For the second proposition, it is plainly false. Foreignizing translation lowers readability, and extending the method to natural contents aggravates such lowering effect to the maximal level possible. It certainly blurs up rather than clarifies the image of the foreign culture, let alone the difference of that and the native culture. How, after all, is a tourist to appreciate the foreignness of an alien behind a blurred wall of glass? Understandably, the motivation behind such a move is to resist forms of cultural hegemony, but rectifying a bad mistake with another bad one is perhaps not the most desirable way out.

\section{PhilosophicAl TransLation}

Different subcategories of translation can face different challenges, for example, literary translation must deal with rhetoric that is deeply involved with the phonological or syntactical features of expressions. In this final section, attention is paid to one issue in philosophical translation, that is, translating philosophical texts, which has been much less discussed compared to literary translations.

The most typical problem in philosophical translation is that certain expressions may have been stipulated (explicitly or implicitly), or "bent", to carry a different meaning than usual, hence already foreignized even to the native readers. Now the translator is faced with one more decision to make: in addition to the choice of how to translate, the translator must also decide which to translate. Should it be the normal expression, leaving the exegetic task to the reader, or should it be the bent expression, building the task of exegesis into translation itself?

This point has been addressed, though against a rather different background, by Peter Long and Roger White in the paper where they proposed "the principle of exegetical neutrality", formulated as "if at any point in a text there is a passage that raises for the native speaker legitimate questions of exegesis, then, if at all possible, a translator should strive to confront the reader of his version with the same questions of exegesis and not produce a version which in his mind resolves those questions." 21 Simply put, the translator should leave the exegetic burden to the reader. As Long \& White (1980) argues (in points 2, 3 and 8 in particular) that exegetically neutral translation best preserves the cross-discourse links: continuity within one writer, the focal point of debates between this writer and academic colleagues, and the reference relation between this writer and secondary works whose opinions differ from one another. If the translator submits an already explained version, the expression in these different texts would come in many versions that appear disconnected, leading to potential confusion.

Here, three more arguments are offered in favor of exegetically neutral translation, in the order of increasing argumentative force. First, if one translates the expression as it is already bent, then many of the metalinguistic sentences are immediately invalidated. Take the classical Chinese term “道”, which literally means road, way, path. In much of Chinese philosophizing, especially in Daoism, this term signifies a multitude of notions such as the ultimate reality, the state of being wise, among others. If one translates it as it is already bent, say, with "logos", "truth", or "wisdom", then sentences such as "The word “道' denotes something rather ordinary and concrete in everyday life" and the like would cease to make sense.

Second, most of the time, the technical expressions are intended by the author to retain a connection with what it ordinarily means, so that a metaphor is built-in in every occurrence of the term. Many of the features that roads (especially big and wide ones) have provide a pictorial trigger for the reader to understand what the more philosophically profound and less linguistically articulable may "look" like. If one translates “道” as explained, the concrete built-in guidance of the abstract concept gets lost.

Third, and worst news for translators who wish to undertake the task of exegesis while translating, is: what if the exegesis turns out misguided, which is unavoidable and indeed rather frequent in cross-cultural communication. There are good reasons to question whether "道” really amounts to what the west has traditionally understood as logos, truth, or wisdom, and the room for open question should not be stuffed by translation, making this move another case of forced ontological mapping, which has proved undesirable.

\section{CONCLUDING REMARKS}

Globalization has found clear expression in the formulation of regional and global markets, but a process of the same name but in a much more fundamental sense is to yet be expected: a meaningful communication and reciprocal

\footnotetext{
${ }^{21}$ Long \& White (1980). The paper concentrates on the translation of one word only, namely, Frege's "Bedeutung", so part of their discussion suits to this specific case alone; the principle was cited to support their choice of translating the word with the natural English counterpart "meaning", rather than argued for in its own right. Beaney (forthcoming) offers a detailed history of how the word has been translated and a criticism of Long and White's choice in this specific case.
} 
enrichment at the level of art and philosophy from different traditions. Good translators seem more needed than ever; they are the quiet but strong, taciturn but eloquent, peaceful but powerful heroes of the epoch, one of tremendous intellectual heritages passed down through history, enveloped in and squeezing against the boundaries of languages, longing for the wider audience outside who are equally longing for them.

It is therefore well worth expecting that more excellent scholars join this historical enterprise, in a way that is best captured in Ames (2017)'s title: better late than never.

\section{REFERENCES}

[1] Ames, Roger. (2017). Better late than never: understanding Chinese philosophy and 'translating it' into the western academy. Ethics and Education. DOI: 10.1080/17449642.2016.1270509.

[2] Beaney, Michael. (forthcoming). Translating "Bedeutung" in Frege's Writings. In Ebert, Philip \& Rossberg, Marcus (Eds.) (forthcoming), Essays on Frege's Basic Laws of Arithmetic. Oxford: Oxford University Press.

[3] Beaney, Michael. (1997). Introduction. In Beaney, Michael (Eds.) (1997), The Frege Reader. Oxford: Blackwell Publishers, $1-46$.

[4] Forster, Michael. (2010). After Herder. Oxford: Oxford University Press.

[5] Giles, Herbert. (1889). Chuang Tzŭ: Mystic, Moralist and Social Reformer. London: Bernard Quaritch Graham.

[6] Hansen, Chad. (1992). A Daoist Theory of Chinese Thought. New York: Oxford University Press.

[7] Long, Peter \& White, Roger. (1980). On the Translation of Frege's Bedeutung: A Reply to Dr Bell. Analysis 40, $196-202$.

[8] Schleiermacher, Friedrich. (1813). On the Different Methods of Translation. (trans. André Lefevere). In A. Leslie Wilson (Eds.) (1982), German Romantic Criticism. New York: Continuum, 1-30.

[9] Venuti, Lawrence. (1995). The Translator's Invisibility. London and New York: Routledge.

[10] Weissbort, Daniel \& Astradur Eysteinsson. (2006). Translation - Theory and Practice. New York: Oxford University Press.

[11] Yan, Fu. (1931). Notes on Translation. In Yan, Fu (Trans.) (1931), Tianyan Lun. Beijing: Commercial Press.

Xinkan Zhao was born in Hangzhou, China. He received his MA degree in philosophy from Peking University, China in 2013.

$\mathrm{He}$ is currently research PhD student in the Department of Philosophy, Peking University, Beijing, China. His research interests include metaethics (with necessary digressions into metaphysics and philosophy of language) and Chinese-to-English philosophical translation. 